\title{
TOEPLITZ OPERATORS WITH BMO SYMBOLS AND THE BEREZIN TRANSFORM
}

\author{
NINA ZORBOSKA
}

Received 3 December 2002

\begin{abstract}
We prove that the boundedness and compactness of the Toeplitz operator on the Bergman space with a $\mathrm{BMO}^{1}$ symbol is completely determined by the boundary behaviour of its Berezin transform. This result extends the known results in the cases when the symbol is either a positive $L^{1}$-function or an $L^{\infty}$ function.
\end{abstract}

2000 Mathematics Subject Classification: 47B35, 32A37.

1. Introduction. Toeplitz operators are one of the most widely studied classes of concrete operators. The study of their behavior on the Hardy and Bergman spaces has generated an extensive list of results in the operator theory and in the theory of function spaces. One of the latest approaches in this area is the use of the Berezin transform as a determining factor of the behaviour of the Toeplitz operator (see $[1,2,6,8,10]$ ). This method is motivated by its connections with quantum physics and noncommutative geometry.

We start with a few of the basic definitions. For more details and references, see $[3,9]$.

The Bergman space $L_{a}^{2}(\mathbb{D})$ is the subspace of $L^{2}(\mathbb{D})$ consisting of functions that are analytic on the unit disk $\mathbb{D}$. Let $P$ be the Bergman projection, that is, the projection form $L^{2}(\mathbb{D})$ onto $L_{a}^{2}(\mathbb{D})$ defined by

$$
(P g)(z)=\int_{\mathbb{D}} \frac{g(\omega)}{(1-z \bar{\omega})^{2}} d m(\omega)
$$

where $d m$ denotes the normalized Lebesque area measure of $\mathbb{D}$. For a function $f$ in $L^{1}(\mathbb{D})$, the Toeplitz operator $T_{f}$ on $L_{a}^{2}(\mathbb{D})$ is defined by

$$
T_{f} \mathcal{g}=P(f g)
$$

Since the Bergman projection kernel function $P$ can be extended to $L^{1}(\mathbb{D})$, the operator $T_{f}$ is well defined on $H^{\infty}$, the space of bounded analytic functions on $\mathbb{D}$. Hence, $T_{f}$ is always densely defined on $L_{a}^{2}(\mathbb{D})$. Since $P$ is not bounded on $L^{1}(\mathbb{D}), T_{f}$ can be unbounded in general. 
For $z$ in $\mathbb{D}$, the Bergman kernel function $K_{z}$ and the normalized Bergman kernel function $k_{z}$ are functions in $L_{a}^{2}(\mathbb{D})$, defined by

$$
K_{z}(\omega)=\frac{1}{(1-\bar{z} \omega)^{2}}, \quad k_{z}(\omega)=\frac{1-|z|^{2}}{(1-\bar{z} \omega)^{2}} .
$$

We have that, for any $g$ in $L_{a}^{2}(\mathbb{D})$,

$$
\left\langle g, K_{z}\right\rangle=g(z) .
$$

Also, $k_{z}$ is in $H^{\infty}$ and $\left\|k_{z}\right\|_{2}=1$, where $\langle\cdot, \cdot\rangle$ denotes the inner product in $L^{2}(\mathbb{D})$ and $\|\cdot\|_{2}$ denotes the $L^{2}(\mathbb{D})$ norm.

For an operator $A$ on $L_{a}^{2}(\mathbb{D})$ that is well defined on $H^{\infty}$, the Berezin transform of $A$ is the function $\tilde{A}$ on $\mathbb{D}$ defined by

$$
\widetilde{A}(z)=\left\langle A k_{z}, k_{z}\right\rangle \text {. }
$$

If $A$ is bounded, then $\widetilde{A}$ is a bounded function. Since the kernels $k_{z}$ converge weakly to zero as $z$ approaches the unit circle $\partial \mathbb{D}$, we have that if $A$ is compact, then $\widetilde{A}(z) \rightarrow 0$ as $z \rightarrow \partial \mathbb{D}$. The converse (in both cases) is not necessarily true and we will mention some counterexamples later on. For $f$ in $L^{1}(\mathbb{D})$, we define the Berezin transform of $f$ to be the function $\tilde{T}_{f}$, that is,

$$
\tilde{f}(z)=\tilde{T}_{f}(z)=\int_{\mathbb{D}} f(\omega)\left|k_{z}(\omega)\right|^{2} d m(\omega) .
$$

Our main result states that for $f$ in the space $\mathrm{BMO}^{1}(\mathbb{D})$ (to be defined later), $T_{f}$ is bounded if and only if $\tilde{f}$ is bounded and $T_{f}$ is compact if and only if $\tilde{f}(z) \rightarrow 0$ as $z \rightarrow \partial \mathbb{D}$. The same result has been proven for positive $L^{1}(\mathbb{D})$ symbols $f$ by Luecking and Zhu in [5, 8], and for $L^{\infty}(\mathbb{D})$ symbols $f$ by Axler and Zheng in [1]. We will see that both of these classes of symbols are contained in $\mathrm{BMO}^{1}(\mathbb{D})$ and so our result covers the above two cases.

We mention few more properties of the Berezin transform function (more details can be found in [1, 3, 9]).

(1) The map $A \rightarrow \tilde{A}$ is one to one.

(2) The function $\tilde{A}(z)$ is in $C^{\infty}(\mathbb{D})$. More precisely, $\tilde{A}(z)$ is real analytic on $\mathbb{D}$ with a power series expansion

$$
\tilde{A}(z)=\left(1-|z|^{2}\right)^{2} \sum_{m, n=0}^{\infty}(m+1)(n+1)\left\langle A z^{n}, z^{m}\right\rangle z^{n} \bar{z}^{m} .
$$

(3) For $f$ in $L^{1}(\mathbb{D}), f$ is harmonic on $\mathbb{D}$ if and only if $f=\tilde{f}$.

While (1) and (2) are fairly easy to obtain, property (3) is a very deep result that was an open conjecture for a number of years, until it was proved independently by Ahern, Flores, and Rudin in 1993 and by English in 1994. For detailed references, see [3]. 
There are several examples in the literature of noncompact operators with Berezin transform vanishing at the boundary (see, e.g., [1]). We mention one of them and then give an example of an unbounded operator with a bounded Berezin transform. Both of the operators will be radial operators, that is, operators that are diagonal with respect to the standard basis $\left\{e_{n}\right\}$ of $L_{a}^{2}(\mathbb{D})$, where $e_{n}(z)=\sqrt{n+1} z^{n}$ (see [10] for more details on radial operators and their Berezin transform).

EXAMPLE 1.1 (see [1, page 392]). Let $A$ be the diagonal operator on $L_{a}^{2}(\mathbb{D})$ defined by

$$
A e_{n}= \begin{cases}0, & n \neq 2^{m} \\ 1, & n=2^{m}\end{cases}
$$

where $m \in \mathbb{N}$. Then $\tilde{A}(z)=\left(1-|z|^{2}\right)^{2} \sum_{m=1}^{\infty}\left(2^{m}+1\right)\left(|z|^{2}\right)^{2^{m}} \rightarrow 0$ as $z \rightarrow \partial \mathbb{D}$, but $A$ is not compact since it is a projection on an infinite-dimensional subspace of $L_{a}^{2}(\mathbb{D})$.

EXAMPLE 1.2. Let $A$ be the diagonal operator on $L_{a}^{2}(\mathbb{D})$ defined by

$$
A e_{n}= \begin{cases}0, & n \neq 2^{m}, \\ m, & n=2^{m},\end{cases}
$$

where $m \in \mathbb{N}$. The operator $A$ is unbounded on $L_{a}^{2}(\mathbb{D})$. We will show that

$$
\tilde{A}(z)=\left(1-|z|^{2}\right)^{2} \sum_{m=1}^{\infty} m\left(2^{m}+1\right)\left(|z|^{2}\right)^{2^{m}}
$$

is bounded on $\mathbb{D}$.

Since $2^{m}+1 \leq 3 \cdot 2^{m-1}$ and $2^{m-1}=\sum_{k=2^{m-1}+1}^{2^{m}} 1$ for all $m$ in $\mathbb{N}$, we have that

$$
\begin{aligned}
\sum_{m=1}^{\infty} m\left(2^{m}+1\right)\left(|z|^{2}\right)^{2^{m}} & \leq 3 \sum_{m=1}^{\infty} m 2^{m-1}\left(|z|^{2}\right)^{2^{m}} \\
& =3 \sum_{m=1}^{\infty} \sum_{k=2^{m-1}+1}^{2^{m}} m\left(|z|^{2}\right)^{2^{m}} \\
& \leq 3 \sum_{m=1}^{\infty} \sum_{k=2^{m-1}+1}^{2^{m}}(k+1)\left(|z|^{2}\right)^{k} \\
& =3 \frac{2|z|^{2}}{\left(1-|z|^{2}\right)^{2}} .
\end{aligned}
$$

Thus $\tilde{A}(z) \leq 6|z|^{2} \leq 6$ for all $z$ in $\mathbb{D}$.

Note that none of the operators in Examples 1.1 and 1.2 is a Toeplitz operator with symbol in $L^{1}(\mathbb{D})$ (see [10] for details). 
We will define the $\mathrm{BMO}^{1}(\mathbb{D})$ spaces for $p \geq 1$ in Section 2 and we will describe some of the properties of the functions belonging to these spaces. The proof of our main result will be presented in Section 3.

Throughout the paper, we will use the letter $c$ to denote a generic positive constant that can change its value at each occurrence.

2. $\mathrm{BMO}^{p}$ spaces. Let $f \in L^{1}(\mathbb{D})$ and let $\psi_{z}$ denote the disk automorphism defined by

$$
\psi_{z}(\omega)=\frac{z-\omega}{1-\bar{z} \omega}
$$

For $p \geq 1$, we say that $f$ belongs to $\mathrm{BMO}^{p}(\mathbb{D})$ whenever

$$
\sup \left\|f \circ \psi_{z}-\tilde{f}(z)\right\|_{p}<\infty
$$

where $\|\cdot\|_{p}$ denotes the $L^{p}(\mathbb{D})$ norm, and $\tilde{f}$ is the Berezin transform of $f$. We define

$$
\begin{gathered}
\|f\|_{\mathrm{BMO}^{p}}=\sup _{z \in \mathbb{D}}\left\|f \circ \psi_{z}-\tilde{f}(z)\right\|_{p}, \\
|\|f\||_{p}=\|f\|_{\mathrm{BMO}^{p}+|\tilde{f}(0)| .}
\end{gathered}
$$

Note that $\|\cdot\|_{\mathrm{BMO}^{p}}$ does not distinguish constants, while $\mid\|\cdot\| \|_{p}$ is a norm in $\mathrm{BMO}^{p}(\mathbb{D})$.

$\mathrm{BMO}^{p}(\mathbb{D})$ spaces were introduced for $p=2$ by Békollé et al. (see [2]) and for general $p \geq 1$ (and general pseudoconvex domains) by Li and Luecking in [4]. There are several equivalent norms on $\mathrm{BMO}^{p}(\mathbb{D})$ that appear in the literature. We will mention another definition stemming from the traditional approach to the BMO spaces on the unit circle. This definition gives a geometric view of the $\mathrm{BMO}^{p}(\mathbb{D})$ spaces by explicitly using the Bergman metric.

For $z, \omega$ in $\mathbb{D}$, let $\beta(z, \omega)=(1 / 2) \log \left(\left(1+\left|\psi_{z}(\omega)\right|\right) /\left(1-\left|\psi_{z}(\omega)\right|\right)\right)$ be the Bergman metric on $\mathbb{D}$. Let $D(z)=\{\omega \in \mathbb{D} ; \beta(z, \omega)<1 / 2\}$ be the Bergman metric disk (also called hyperbolic disk) with center $\mathrm{z}$ and radius $1 / 2$. The normalized area of $D(z)$, denoted by $|D(z)|$, is equivalent to $1 /\left(1-|z|^{2}\right)^{2}$. For more details, see [3, 9].

For $f$ in $L^{1}(\mathbb{D})$, the average of $f$ over $D(z)$ is defined by

$$
\hat{f}(z)=\frac{1}{|D(z)|} \int_{D(z)} f(\omega) d m(\omega) .
$$

Using properties of the Bergman metric and results from [4], it follows that $\|f\|_{\mathrm{BMO}^{p}}$ is finite if and only if

$$
\sup _{z \in \mathbb{D}} \frac{1}{|D(z)|} \int_{D(z)}|f(\omega)-\hat{f}(z)|^{p} d m(\omega)<\infty
$$


Thus, functions in $\mathrm{BMO}^{p}(\mathbb{D})$ have bounded mean oscillation in the Bergman metric. Note that any other choice for the radius of the hyperbolic disk $D(z)$ gives the same set of functions. Since $\mathrm{BMO}^{p}(\mathbb{D})$ functions are locally in $L^{p}(\mathbb{D})$, the spaces are different for different $p$. It is not hard to see that

$$
\begin{gathered}
L^{\infty}(\mathbb{D}) \subset \mathrm{BMO}^{p}(\mathbb{D}) \subset L^{p}(\mathbb{D}), \quad \text { for } p \geq 1, \\
\mathrm{BMO}^{q}(\mathbb{D}) \subset \mathrm{BMO}^{p}(\mathbb{D}) \subset \mathrm{BMO}^{1}(\mathbb{D}), \quad \text { for } 1 \leq p<q .
\end{gathered}
$$

Details regarding (2.6) could be found in $[4,7,9]$.

Since the space $\mathrm{BMO}^{1}(\mathbb{D})$ is the largest among the $\mathrm{BMO}^{p}(\mathbb{D})$ spaces for $p \geq 1$, from now on we will be mainly interested in functions belonging to this class. We will also drop the reference to the unit disk and simply write $\mathrm{BMO}^{p}$ instead of $\mathrm{BMO}^{p}(\mathbb{D})$.

The next proposition says more about the Berezin transform of $\mathrm{BMO}^{1}$ functions. Similar properties have been proven about $\mathrm{BMO}^{2}$ functions (see [2]).

Proposition 2.1. Let $f$ be in $\mathrm{BMO}^{1}$. Then

(a) $\sup _{z \in \mathbb{D}}(\widetilde{|f|}(z)-|\tilde{f}(z)|)<\infty$,

(b) $\tilde{f}$ is Lipschitz with respect to the Bergman metric,

(c) $\sup _{z \in \mathbb{D}}\left(1-|z|^{2}\right)|\nabla \tilde{f}(z)|<\infty$,

(d) $\sup _{z \in \mathbb{D}}|\widetilde{f-\tilde{f}}|(z)<\infty$.

Proof. (a) For $z$ in $\mathbb{D}$, we have that

$$
\begin{aligned}
(\widetilde{|f|}(z)-|\tilde{f}(z)|) & =\int_{\mathbb{D}}(|f(\omega)|-|\tilde{f}(z)|)\left|k_{z}(\omega)\right|^{2} d m(\omega) \\
& \leq \int_{\mathbb{D}}|f(\omega)-\tilde{f}(z)|\left|k_{z}(\omega)\right|^{2} d m(\omega) \\
& =\int_{\mathbb{D}}\left|f \circ \psi_{z}(v)-\tilde{f}(z)\right| d m(v) \\
& =\left\|f \circ \psi_{z}(v)-\tilde{f}(z)\right\|_{1},
\end{aligned}
$$

where we have used the change of variable $\omega=\psi_{z}(v)$ in the third line of (2.7).

Since $f \in \mathrm{BMO}^{1}, \sup _{z \in \mathbb{D}}\left\|f \circ \psi_{z}(v)-\tilde{f}(z)\right\|_{1}<\infty$ and so $\sup _{z \in \mathbb{D}}(\widetilde{|f|}(z)-$ $|\tilde{f}(z)|)<\infty$ as well.

(b) We have to show that there exists a constant $c>0$ such that, for every $z, \omega \in \mathbb{D}$,

$$
|\tilde{f}(z)-\tilde{f}(\omega)| \leq c \beta(z, \omega)
$$

Békollé et al. have established in [2] that the same property for $\tilde{f}$ is true in the case when $f$ belongs to $\mathrm{BMO}^{2}$. We will explain the main idea of the proof and the part where our proof differs from that in [2].

For $z, \omega \in \mathbb{D}$, let $\alpha(t)$ denote the geodesic from $z=\alpha(0)$ to $\omega=\alpha(1)$ in the Bergman metric, and let $s=s(t)$ denote the arc length of $\alpha(t)$ in the Bergman 
metric. Since

$$
|\tilde{f}(z)-\tilde{f}(\omega)| \leq \int_{0}^{1}\left|\frac{d}{d t}(\tilde{f}(\alpha(t)))\right| d t
$$

it suffices to estimate $(d / d t)(\tilde{f}(\alpha(t)))$, for $f$ in $\mathrm{BMO}^{1}$.

The following inequality can be found in [3, page 48] and is a part of the above-mentioned proof in [2] that we use here:

$$
\begin{aligned}
& \left|\frac{d}{d t}(\tilde{f}(\alpha(t)))\right| \\
& \quad \leq 4 \int_{\mathbb{D}}|f(\omega)-\tilde{f}(\alpha(t))|\left|k_{\alpha(t)}(\omega)\right| \frac{\left|\alpha^{\prime}(t)\right||\omega-\alpha(t)|}{|1-\overline{\alpha(t)} \omega|^{3}} d m(\omega) .
\end{aligned}
$$

Since

$$
\frac{\left|\alpha^{\prime}(t)\right|}{1-|\alpha(t)|^{2}}=\frac{d s}{d t}
$$

and since

$$
\frac{|\omega-\alpha(t)|}{|1-\overline{\alpha(t)} \omega|}=\left|\psi_{\alpha(t)}(\omega)\right| \leq 1
$$

we get that

$$
\begin{aligned}
\frac{\left|\alpha^{\prime}(t)\right||\omega-\alpha(t)|}{|1-\overline{\alpha(t)} \omega|^{3}} & =\frac{\left|\alpha^{\prime}(t)\right|}{1-|\alpha(t)|^{2}} \frac{1-|\alpha(t)|^{2}}{|1-\overline{\alpha(t)} \omega|^{2}}\left|\psi_{\alpha(t)}(\omega)\right| \\
& \leq \frac{d s}{d t}\left|k_{\alpha(t)}(\omega)\right| .
\end{aligned}
$$

Hence,

$$
\begin{aligned}
\left|\frac{d}{d t}(\tilde{f}(\alpha(t)))\right| & \leq 4 \frac{d s}{d t} \int_{\mathbb{D}}|f(\omega)-\tilde{f}(\alpha(t))|\left|k_{\alpha(t)}(\omega)\right|^{2} d m(\omega) \\
& =4 \frac{d s}{d t}\left\|f \circ \psi_{\alpha(t)}-\tilde{f}(\alpha(t))\right\|_{1} \\
& \leq 4 \frac{d s}{d t}\|f\|_{\mathrm{BMO}^{1}} .
\end{aligned}
$$

So,

$$
|\tilde{f}(z)-\tilde{f}(\omega)| \leq 4\|f\|_{\mathrm{BMO}^{1}} \int_{\mathbb{D}} \frac{d s}{d t} d t=4\|f\|_{\mathrm{BMO}^{1}} \beta(z, \omega),
$$

and the constant $c$ can be chosen to be equal to $4\|f\|_{\mathrm{BMO}^{1}}$.

(c) We will show that $|\nabla \tilde{f}(z)| \leq c /\left(1-|z|^{2}\right)$, where $\nabla \tilde{f}(z)$ is the complex vector $(\partial \tilde{f} / \partial x, \partial \tilde{f} / \partial y)$ for $z=x+i y$ and

$$
|\nabla \tilde{f}(z)|^{2}=\left|\frac{\partial \tilde{f}}{\partial x}\right|^{2}+\left|\frac{\partial \tilde{f}}{\partial y}\right|^{2} .
$$


Using the fact that $\lim _{h \rightarrow 0}(\beta(z+h, z) /|h|)=1 /\left(1-|z|^{2}\right)$ and part (b), we get that

$$
\begin{aligned}
\left|\frac{\partial}{\partial x} \tilde{f}(z)\right| & \leq \lim _{h \rightarrow 0} \frac{|\tilde{f}(x+h+i y)-\tilde{f}(x+i y)|}{|h|} \\
& \leq c \lim _{h \rightarrow 0} \frac{\beta(x+h+i y, x+i y)}{|h|}=\frac{c}{1-|z|^{2}} .
\end{aligned}
$$

Similarly,

$$
\left|\frac{\partial}{\partial y} \tilde{f}(z)\right| \leq \frac{c}{1-|z|^{2}}
$$

and so $|\nabla \tilde{f}(z)|^{2} \leq 2\left(c^{2} /\left(1-|z|^{2}\right)^{2}\right)$.

(d) Since $|f-\tilde{f}| \geq 0$, by [8] we have that $\widetilde{f-\tilde{f}} \mid$ is bounded if and only if $|\widehat{f-\tilde{f}}|$ is bounded. Using part (b) and the fact that for $\omega$ in $D(z), 1 /|D(z)| \sim$ $\left|k_{z}(\omega)\right|^{2}$, we get that

$$
\begin{aligned}
|\widehat{f-\tilde{f}}|(z)= & \frac{1}{|D(z)|} \int_{D(z)}|f(\omega)-\tilde{f}(\omega)| d m(\omega) \\
\leq & \frac{1}{|D(z)|} \int_{D(z)}|f(\omega)-\tilde{f}(z)| d m(\omega) \\
& +\frac{1}{|D(z)|} \int_{D(z)}|f(z)-\tilde{f}(\omega)| d m(\omega) \\
\leq & c \int_{\mathbb{D}}|f(\omega)-\tilde{f}(z)|\left|k_{z}(\omega)\right|^{2} d m(\omega) \\
& +c \frac{1}{|D(z)|} \int_{D(z)} \beta(z, \omega) d m(\omega) \\
\leq & c\left\|f \circ \psi_{z}-\tilde{f}(z)\right\|_{1}+\frac{1}{2} c<\infty
\end{aligned}
$$

since $\sup _{z \in \mathbb{D}}\left\|f \circ \psi_{z}-\tilde{f}(z)\right\|_{1}<\infty$.

Proposition 2.2. Let $f$ be in $L^{1}(\mathbb{D})$.

(a) Let $\tilde{f}$ be bounded in $\mathbb{D}$. Then $\sup _{z \in \mathbb{D}}(\widetilde{|f|}(z)-|\tilde{f}(z)|)<\infty$ implies that $f$ is in $\mathrm{BMO}^{1}$.

(b) There exists $c \geq 0$ such that $(\hat{f}(z)-\tilde{f}(z)) \leq c\left\|f \circ \psi_{z}-\tilde{f}(z)\right\|_{1}$ for every $z$ in $\mathbb{D}$.

Proof. (a) Whenever $\tilde{f}$ is bounded and $\sup _{z \in \mathbb{D}}(|\widetilde{f}|(z)-|\tilde{f}(z)|)<\infty$, we have that $\widetilde{|f|}$ is also bounded. But, since

$$
\left\|f \circ \psi_{z}-\tilde{f}(z)\right\|_{1} \leq\left\|f \circ \psi_{z}\right\|_{1}+|\tilde{f}(z)|=\widetilde{|f|}(z)+|\tilde{f}(z)|,
$$

we get that $\sup _{z \in \mathbb{D}}\left\|f \circ \psi_{z}-\tilde{f}(z)\right\|_{1}<\infty$ and thus $f$ is in $\mathrm{BMO}^{1}$. 
(b) Using the fact that for $\omega$ in $D(z)$ we have that $1 /|D(z)| \leq c\left|k_{z}(\omega)\right|^{2}$, it follows that

$$
\begin{aligned}
|\hat{f}(z)-\tilde{f}(z)| & \leq \frac{1}{|D(z)|} \int_{D(z)}|f(\omega)-\tilde{f}(z)| d m(\omega) \\
& \leq c \int_{D(z)}|f(\omega)-\tilde{f}(z)|\left|k_{z}(\omega)\right|^{2} d m(\omega) \\
& \leq c\left\|f \circ \psi_{z}-\tilde{f}(z)\right\|_{1} .
\end{aligned}
$$

As a consequence of the results in Propositions 2.1 and 2.2, we get the following corollary.

Corollary 2.3. Let $f$ be in $L^{1}(\mathbb{D})$.

(a) For $f$ in $\mathrm{BMO}^{1}, \tilde{f}$ being bounded implies that $T_{f}$ is bounded on $L_{a}^{2}(\mathbb{D})$.

(b) Every $f$ in $\mathrm{BMO}^{1}$ can be written as $f=f_{1}+f_{2}$ with $f_{1}$ in the (real) Bloch space and $f_{2}$ such that $\sup _{z \in \mathbb{D}} \mid \widetilde{f_{2} \mid}(z)<\infty$.

(c) If $f \geq 0$ on $\mathbb{D}$ and if $\widetilde{f}$ is bounded, then $f$ belongs to $\mathrm{BMO}^{1}$.

(d) For $f$ in $\mathrm{BMO}^{1}, \tilde{f}$ is bounded if and only if $\hat{f}$ is bounded.

Proof. (a) Using the fact that for every $f$ in $L^{1}(\mathbb{D})$ we have

$$
\begin{aligned}
|\tilde{f}(z)| & =\left.\left|\int_{\mathbb{D}} f(\omega)\right| k_{z}(\omega)\right|^{2} d m(\omega) \mid \\
& \leq \int_{\mathbb{D}} f(\omega)\left|k_{z}(\omega)\right|^{2} d m(\omega)=\widetilde{|f|}(z),
\end{aligned}
$$

it follows from Proposition 2.1(a) that whenever $f$ is in $\mathrm{BMO}^{1}$ and $\tilde{f}$ is bounded, we have that $\widetilde{|f|}$ is also bounded. Since $|f| \geq 0$, by results from $[5,8], \widetilde{f} \mid$ being bounded implies that $T_{|f|}$ is bounded. Then it is not hard to see that $T_{f}$ has to be bounded too.

(b) Take $f_{1}=\tilde{f}$ and $f_{2}=f-\tilde{f}$. Then the rest follows from Proposition 2.1(c) and (d).

(c) For $f \geq 0$, we have $\widetilde{\mid f}|(z)=| \tilde{f} \mid(z)=\tilde{f}(z)$. In case $\tilde{f}$ is bounded, using Proposition 2.2(a), we get that $f$ belongs to $\mathrm{BMO}^{1}$.

(d) It follows directly from Proposition 2.2(b).

3. Proof of the main theorem. Our main theorem (Theorem 3.1) expands the class of functions $f$ for which it is known that $\tilde{f}(z) \rightarrow 0$, as $z \rightarrow \partial \mathbb{D}$ implies that $T_{f}$ is compact. It includes $L^{\infty}$ functions and positive $L^{1}$ functions with bounded Berezin transform, and so the theorem is an extension of the results of Axler and Zheng (see [1]) and Luecking and Zhu (see [5, 8]).

THEOREM 3.1. Let $f$ belong to $\mathrm{BMO}^{1}$. Then $\tilde{f}(z) \rightarrow 0$, as $z \rightarrow \partial \mathbb{D}$ implies that $T_{f}$ is compact on $L_{a}^{2}(\mathbb{D})$.

Before we proceed with the proof, we state two lemmas that contain some of the more technical parts used in the proof. 
LEMMA 3.2. Let $f$ be in $L^{1}(\mathbb{D})$ and let $T_{f}$ be bounded on $L_{a}^{2}(\mathbb{D})$. Then for every $z$ in $\mathbb{D}$, the following is true:

(a) $\left(T_{f} K_{z}\right)(u)=K_{z}(u) P\left(f \circ \psi_{z}\right)\left(\psi_{z}(u)\right)$,

(b) $\left\|T_{f} k_{z}\right\|_{2}=\left\|T_{f \circ \psi_{z}} \mathbf{1}\right\|_{2}$,

(c) for $f$ in $\mathrm{BMO}^{1}$, each $T_{f \circ \psi_{z}}$ is bounded on $L_{a}^{2}(\mathbb{D})$.

Proof. (a) We have the following equalities:

$$
\begin{aligned}
\left(T_{f} K_{z}\right)(u) & =\left\langle P\left(f K_{z}\right), K_{u}\right\rangle \\
& =\int_{\mathbb{D}} f(\omega) K_{z}(\omega) \overline{K_{u}(\omega)} d m(\omega) \\
& =\int_{\mathbb{D}} f\left(\psi_{z}(v)\right) K_{z}\left(\psi_{z}(v)\right) \overline{K_{u}\left(\psi_{z}(v)\right)}\left|k_{z}(v)\right|^{2} d m(v),
\end{aligned}
$$

where we have used the change of variable $\omega=\psi_{z}(v)$, and the fact that $\left|\psi_{z}^{\prime}(v)\right|=\left|k_{z}(v)\right|$.

Using the equations

$$
\begin{aligned}
& K_{z}\left(\psi_{z}(v)\right) k_{z}(v)=\frac{1}{1-|z|^{2}}, \\
& \overline{K_{u}\left(\psi_{z}(v)\right) k_{z}(v)}=k_{z}(v) \overline{K_{\psi_{z}(u)}(v)}
\end{aligned}
$$

(which can be checked directly from the definitions of the functions involved), we get that

$$
\begin{aligned}
\left(T_{f} K_{z}\right)(u) & =\int_{\mathbb{D}}\left(f \circ \psi_{z}\right)(v) \frac{1}{1-|z|^{2}} k_{z}(u) \overline{K_{\psi_{z}(u)}(v)} d m(v) \\
& =K_{z}(u) \int_{\mathbb{D}}\left(f \circ \psi_{z}\right)(v) \overline{K_{\psi_{z}(u)}(v)} d m(v) \\
& =K_{z}(u) P\left(f \circ \psi_{z}\right)\left(\psi_{z}(u)\right) .
\end{aligned}
$$

(b) We have that

$$
\begin{aligned}
\left\|T_{f} k_{z}\right\|_{2}^{2} & =\left\|P\left(f k_{z}\right)\right\|_{2}^{2} \\
& =\int_{\mathbb{D}}\left|P\left(f k_{z}\right)(\omega)\right|^{2} d m(\omega) \\
& =\int_{\mathbb{D}}\left|P\left(f k_{z}\right)\left(\psi_{z}(u)\right)\right|^{2}\left|\psi_{z}^{\prime}(u)\right|^{2} d m(u),
\end{aligned}
$$

by the change of the variable $\omega=\psi_{z}(u)$. Using the fact that

$$
\overline{K_{\psi_{z}(u)}(\omega)} \psi_{z}^{\prime}(u)=\overline{k_{z}(\omega) K_{u}\left(\psi_{z}(\omega)\right)}
$$


we can continue with the following equations:

$$
\begin{aligned}
\int_{\mathbb{D}} \mid & \left.P\left(f k_{z}\right)\left(\psi_{z}(u)\right)\right|^{2}\left|\psi_{z}^{\prime}(u)\right|^{2} d m(u) \\
& =\int_{\mathbb{D}}\left|\int_{\mathbb{D}} f(\omega) k_{z}(\omega) \overline{K_{\psi_{z}(u)}(\omega)} \psi_{z}^{\prime}(u) d m(\omega)\right|^{2} d m(u) \\
& =\int_{\mathbb{D}}\left|\int_{\mathbb{D}} f(\omega) k_{z}(\omega) \overline{k_{z}(\omega)} \overline{K_{u}\left(\psi_{z}(\omega)\right)} d m(\omega)\right|^{2} d m(u) \\
& =\left.\left.\int_{\mathbb{D}}\left|\int_{\mathbb{D}} f(\omega)\right| k_{z}(\omega)\right|^{2} \overline{K_{u}\left(\psi_{z}(\omega)\right)} d m(\omega)\right|^{2} d m(u) \\
& =\int_{\mathbb{D}}\left|\int_{\mathbb{D}} f\left(\psi_{z}(v)\right) \overline{K_{u}(v)} d m(v)\right|^{2} d m(u) \\
& =\int_{\mathbb{D}}\left|P\left(f \circ \psi_{z}\right)(u)\right|^{2} d m(u) \\
& =\left\|P\left(f \circ \psi_{z}\right)\right\|_{2}^{2}=\left\|T_{f \circ \psi_{z}} \mathbf{1}\right\|_{2}^{2},
\end{aligned}
$$

where we have used the change of the variable $v=\psi_{z}(\omega)$ in the fourth equation. Thus $\left\|T_{f} k_{z}\right\|_{2}^{2}=\left\|T_{f \circ \psi_{z}} \mathbf{1}\right\|_{2}^{2}$.

(c) It follows from the definition of $\mathrm{BMO}^{1}$ that, whenever $f$ belongs to $\mathrm{BMO}^{1}$, $f \circ \psi_{z}$ also belongs to $\mathrm{BMO}^{1}$, for all $z$ in $\mathbb{D}$.

Furthermore,

$$
\begin{aligned}
\widetilde{f \circ \psi_{z}}(\omega) & =\int_{\mathbb{D}} f \circ \psi_{z}(u)\left|k_{\omega}(u)\right|^{2} d m(u) \\
& =\int_{\mathbb{D}} f(v)\left|k_{\omega}\left(\psi_{z}(v)\right)\right|^{2}\left|\psi_{z}^{\prime}(v)\right|^{2} d m(v) \\
& =\int_{\mathbb{D}} f(v)\left|k_{\psi_{z}(\omega)}(v)\right|^{2} d m(v) \\
& =\widetilde{f}\left(\psi_{z}(\omega)\right),
\end{aligned}
$$

(where we have used that $\left|k_{s}(v)\right|^{2}=\left|\psi_{s}^{\prime}(v)\right|^{2}$ and that $\left(\psi_{\omega} \circ \psi_{z}\right)(v)=$ $\psi_{\psi_{z}(\omega)}(v)$ ). Thus, $\tilde{f}$ being bounded implies that $\widetilde{f \circ \psi_{z}}$ is bounded independently of $z$. By Corollary 2.3(a), it follows that for $f$ in $\mathrm{BMO}^{1}$ and $\tilde{f}$ being bounded, each $T_{f \circ \psi_{z}}$ is bounded on $L_{a}^{2}(\mathbb{D})$.

LEMMA 3.3. Let $p$ and $\varepsilon$ be positive numbers such that $p>3$ and $1 / p<\varepsilon<$ $(1 / 2)(1-1 / p)$. Then

$$
\int_{\mathbb{D}} \frac{\left(1-|v|^{2}\right)^{-2 p \varepsilon /(p-1)}}{|1-\bar{z} v|^{2 p(1-2 \varepsilon) /(p-1)}} d m(v)
$$

is bounded in $z$. 
Proof. Let $t=-2 p \varepsilon /(p-1)$ and let $c=-t-2+2 p(1-2 \varepsilon) /(p-1)$. Then, $2 p \varepsilon /(p-1)<(1-1 / p) p /(p-1)=1$ and $c<0$, since

$$
\begin{aligned}
\frac{2 p \varepsilon}{p-1}-2+\frac{2 p(1-2 \varepsilon)}{p-1} & =\frac{2 p \varepsilon-2 p+2+2 p-4 p \varepsilon}{p-1} \\
& =\frac{2-2 p \varepsilon}{p-1}<\frac{2-2 p(1 / p)}{p-1}=0
\end{aligned}
$$

By [9, Lemma 4.2.2], since $t>-1$ and $c<0$, we get that the integral is bounded in $z$.

The proof of the theorem will be done in several steps. The steps follow the standard idea of finding a sequence of compact operators that converges to the given operator. To establish the convergence, we will use the Schur's test. The same approach has also been implemented in $[1,6]$. The core of our extension is contained in the first step of the proof.

PROOF OF THEOREM 3.1

STEP 1. Let $f \in \mathrm{BMO}^{1}$ and let $\tilde{f}$ be bounded. Then

$$
\sup _{z \in \mathbb{D}}\left\|T_{f \circ \psi_{z}} \mathbf{1}\right\|_{p}<\infty, \quad \forall p \geq 1 .
$$

Proof OF STEP 1. For an analytic function $g$, its $L^{p}$ norm $\|g\|_{p}$ is equivalent to $|g(0)|+\left\|\left(1-|z|^{2}\right) g^{\prime}(z)\right\|_{p}$. For details, see, for example, [9].

The Bloch spaces $B$ is defined by

$$
B=\left\{g \text { analytic on } \mathbb{D} ; \sup _{z \in \mathbb{D}}\left(1-|z|^{2}\right)\left|g^{\prime}(z)\right|<\infty\right\} \text {. }
$$

For $g$, being a function in the Bloch space $B$, let $\|g\|_{B}=|g(0)|+\|(1-$ $\left.|z|^{2}\right)\left|g^{\prime}(z)\right| \|_{\infty}$. It follows that

$$
\begin{aligned}
\|g\|_{p} & \leq c\left(|g(0)|+\left\|\left(1-|z|^{2}\right) g^{\prime}(z)\right\|_{p}\right) \\
& \leq c\left(|g(0)|+\left\|\left(1-|z|^{2}\right) g^{\prime}(z)\right\|_{\infty}\right) \\
& =c\|g\|_{B} .
\end{aligned}
$$

It has been proven by Li and Luecking in [4] that the Bergman projection $P$ is a bounded operator from $\mathrm{BMO}^{1}$ into $B$, for all $p \geq 1$. Since, for $f$ in $\mathrm{BMO}^{1}$ we have that $f \circ \psi_{z}$ is in $\mathrm{BMO}^{1}$ for all $z$ in $\mathbb{D}$, we get that $P\left(f \circ \psi_{z}\right) \in B$. Hence

$$
\begin{aligned}
\left\|P\left(f \circ \psi_{z}\right)\right\|_{p} & \leq c\left\|P\left(f \circ \psi_{z}\right)\right\|_{B} \leq c \mid\left\|f \circ \psi_{z}\right\| \|_{1} \\
& =c\left(\left|\widetilde{f \circ \psi_{z}}(0)\right|+\sup _{z \in \mathbb{D}}\left\|f \circ \psi_{z} \circ \psi_{\omega}-\widetilde{f \circ \psi_{z}}(\omega)\right\|_{1}\right)
\end{aligned}
$$




$$
\begin{aligned}
& =c\left(|\tilde{f}(z)|+\sup _{\psi_{z}(\omega) \in \mathbb{D}}\left\|f \circ \psi_{\psi_{z}(\omega)}-\tilde{f}\left(\psi_{z}(\omega)\right)\right\|_{1}\right) \\
& =c\left(|\tilde{f}(z)|+\sup _{u \in \mathbb{D}}\left\|f \circ \psi_{u}-\tilde{f}(u)\right\|_{1}\right) .
\end{aligned}
$$

So, for $f$ in $\mathrm{BMO}^{1}$ and $\tilde{f}$ being bounded, we get that

$$
\sup _{z \in \mathbb{D}}\left\|T_{f \circ \psi_{z}} \mathbf{1}\right\|_{p}=\sup _{z \in \mathbb{D}}\left\|P\left(f \circ \psi_{z}\right)\right\|_{p}<\infty, \quad \forall p \geq 1 .
$$

STEP 2. Let $T_{f}$ be bounded on $L_{a}^{2}(\mathbb{D})$ and let $\tilde{f}(z) \rightarrow 0$ as $z \rightarrow \partial \mathbb{D}$. Then $T_{f \circ \psi_{z}} \mathbf{1} \rightarrow 0$ weakly as $z \rightarrow \partial \mathbb{D}$.

Proof OF STEP 2 (see [1, page 396]). The proof uses the explicit doubleseries form of the Berezin transform of an operator in $L_{a}^{2}(\mathbb{D})$.

We mention that, since the specific nature of the Toeplitz operator is not used in the proof, a more general statement is true.

Let $A$ be a bounded operator on $L_{a}^{2}(\mathbb{D})$ and let $U_{z}$ be the unitary operator on $L_{a}^{2}(\mathbb{D})$, defined by

$$
U_{z} \mathfrak{g}=\left(g \circ \psi_{z}\right) \psi_{z}^{\prime}
$$

Then $\tilde{A}(z) \rightarrow 0$, as $z \rightarrow \partial \mathbb{D}$, implies that $U_{z} A U_{z} \mathbf{1} \rightarrow 0$ weakly as $z \rightarrow \partial \mathbb{D}$.

STEP 3. Let $f \in \mathrm{BMO}^{1}$. Then $\tilde{f}(z) \rightarrow 0$, as $z \rightarrow \partial \mathbb{D}$, implies that $\left\|T_{f \circ \psi_{z}} \mathbf{1}\right\|_{2} \rightarrow$ 0 , as $z \rightarrow \partial \mathbb{D}$.

PROOF OF STEP 3. The method of the proof is similar to a part of the proof in [1]. For the sake of completeness, we provide the details.

By Step 2, $T_{f \circ \psi_{z}} \mathbf{1} \rightarrow 0$ weakly and so it converges uniformly to zero on compact subsets of $\mathbb{D}$, such as $r \overline{\mathbb{D}}$, for $0 \leq r<1$. Since

$$
\begin{aligned}
\left\|T_{f \circ \psi_{z}} \mathbf{1}\right\|_{2}^{2}= & \int_{\mathbb{D}}\left|T_{f \circ \psi_{z}} \mathbf{1}(\omega)\right|^{2} d m(\omega) \\
= & \int_{\mathbb{D} \backslash r \overline{\mathbb{D}}}\left|T_{f \circ \psi_{z}} \mathbf{1}(\omega)\right|^{2} d m(\omega) \\
& +\int_{r \overline{\mathbb{D}}}\left|T_{f \circ \psi_{z}} \mathbf{1}(\omega)\right|^{2} d m(\omega),
\end{aligned}
$$

we also need to estimate the first integral of the last line. We will do that by using the Cauchy-Schwartz inequality and the result of Step 1:

$$
\begin{aligned}
& \int_{\mathbb{D} \backslash r \overline{\mathbb{D}}}\left|T_{f \circ \psi_{z}} \mathbf{1}(\omega)\right|^{2} d m(\omega) \\
& \quad \leq\left(\int_{\mathbb{D} \backslash r \overline{\mathbb{D}}}\left|T_{f \circ \psi_{z}} \mathbf{1}(\omega)\right|^{4} d m(\omega)\right)^{1 / 2}\left(\int_{\mathbb{D} \backslash r \overline{\mathbb{D}}} d m(\omega)\right)^{1 / 2} \\
& \quad \leq\left\|T_{f \circ \psi_{z}} \mathbf{1}\right\|_{4}^{2}\left(1-r^{2}\right)^{1 / 2} \leq c\left(1-r^{2}\right)^{1 / 2} .
\end{aligned}
$$


We can make the integral over $\mathbb{D} \backslash r \overline{\mathbb{D}}$ as small as we wish, independently of $z$, by choosing $r$ close enough to 1 . Then, for the same $r$, take $z$ close enough to $\partial \mathbb{D}$ such that the integral over $r \overline{\mathbb{D}}$ is also as small as we wish.

STEP 4. Let $f \in \mathrm{BMO}^{1}$. Then $\tilde{f}(z) \rightarrow 0$, as $z \rightarrow \partial \mathbb{D}$, implies that $\left\|T_{f \circ \psi_{z}} \mathbf{1}\right\|_{p} \rightarrow 0$ as $z \rightarrow \partial \mathbb{D}$, for all $p \geq 1$.

Proof OF STEP 4. For $p<2$, we have that $\left\|T_{f \circ \psi_{z}} \mathbf{1}\right\|_{p} \leq\left\|T_{f \circ \psi_{z}} \mathbf{1}\right\|_{2}$ and the rest follows from Step 3. For $p>2$,

$$
\left\|T_{f \circ \psi_{z}} \mathbf{1}\right\|_{p}^{p} \leq\left\|T_{f \circ \psi_{z}} \mathbf{1}\right\|_{2}\left\|T_{f \circ \psi_{z}} \mathbf{1}\right\|_{2 p-2}^{p-1}
$$

by Hölder's inequality. The proof follows from Steps 3 and 1 , since $2 p-2>$ $2 \geq 1$.

STEP 5. Let $f \in \mathrm{BMO}^{1}$ and let $\tilde{f}(z) \rightarrow 0$ as $z \rightarrow \partial \mathbb{D}$. For $0<r<1$, let $T_{r}^{f}$ be the operator from $L_{a}^{2}(\mathbb{D})$ into $L^{2}(\mathbb{D})$, defined by

$$
T_{r}^{f}=M_{\chi_{r \mathbb{D}}} T_{f}
$$

where $M_{\chi_{r \mathbb{D}}}$ is the multiplication operator on $L_{a}^{2}(\mathbb{D})$ with $\chi_{r \mathbb{D}}$ being the characteristic function of $r \mathbb{D}$. Let $T^{f}$ denote the operator $T_{f}$ as an operator from $L_{a}^{2}(\mathbb{D})$ into $L^{2}(\mathbb{D})$. Then $T_{r}^{f}$ is compact and $\lim _{r \rightarrow 1}\left\|T^{f}-T_{r}^{f}\right\|=0$.

Proof OF STEP 5. It is well known that the operator $M_{\chi_{r} \mathbb{D}}$ is compact on $L^{2}(\mathbb{D})$ since $\chi_{r \mathbb{D}}(z)=0$ for $|z| \geq r$. Thus, $T_{r}^{f}$ is also compact, as a product of a compact and a bounded operator.

For $g$ in $L_{a}^{2}(\mathbb{D})$, we have that

$$
\begin{aligned}
\left(T^{f}-T_{r}^{f}\right) g(z) & =\left(\left(1-\chi_{r \mathbb{D}}\right) T_{f} g\right)(z) \\
& =\chi_{\mathbb{D} \backslash r \mathbb{D}}(z)\left\langle T_{f} g, K_{z}\right\rangle=\chi_{\mathbb{D} \backslash r \mathbb{D}}(z)\left\langle g, T_{\bar{f}} K_{z}\right\rangle \\
& =\int_{\mathbb{D}} g(u) \chi_{\mathbb{D} \backslash r \mathbb{D}}(z) \overline{T_{\bar{f}} K_{z}}(u) d m(u) .
\end{aligned}
$$

So, $T^{f}-T_{r}^{f}$ is an integral operator with kernel $K_{r}^{f}(z, u)=\chi_{\mathbb{D} \backslash r \mathbb{D}}(z) \overline{T_{\bar{f}} K_{z}}(u)$. By Schur's test, whenever there exist a positive measurable function $h$ on $\mathbb{D}$ and constants $c_{1}$ and $c_{2}$ such that

$$
\begin{aligned}
& \int_{\mathbb{D}}\left|K_{r}^{f}(z, u)\right| h(z) d m(z) \leq c_{1} h(u), \quad \forall u \text { in } \mathbb{D}, \\
& \int_{\mathbb{D}}\left|K_{r}^{f}(z, u)\right| h(u) d m(u) \leq c_{2} h(z), \quad \forall z \text { in } \mathbb{D},
\end{aligned}
$$

we have that $\left\|T^{f}-T_{r}^{f}\right\|^{2} \leq c_{1} c_{2}$. 
Let $p>3,1 / p<\varepsilon<(1 / 2)(1-1 / p)$ and let $h(z)=\left(K_{z}(z)\right)^{\varepsilon}=1 /\left(1-|z|^{2}\right)^{2 \varepsilon}$. We will show that the Schur's test works with constants

$$
c_{1}=c \sup _{z \in \mathbb{D}}\left\|T_{f \circ \psi_{z}} \mathbf{1}\right\|_{p}, \quad c_{2}=c \sup _{|z| \geq r}\left\|T_{\bar{f} \circ \psi_{z}} \mathbf{1}\right\|_{p} .
$$

We have that

$$
\int_{\mathbb{D}}\left|K_{r}^{f}(z, u)\right| h(u) d m(u)=\chi_{\mathbb{D} \backslash r \mathbb{D}}(z) \int_{\mathbb{D}}\left|T_{\bar{f}} K_{z}(u)\right|\left(K_{u}(u)\right)^{\varepsilon} d m(u),
$$

which by Lemma 3.2(a) equals

$$
\chi_{\mathbb{D} \backslash r \mathbb{D}}(z) \int_{\mathbb{D}}\left|K_{z}(u)\right|\left|P\left(\bar{f} \circ \psi_{z}\right)\left(\psi_{z}(u)\right)\right|\left(K_{u}(u)\right)^{\varepsilon} d m(u) .
$$

By the change of variable $\psi_{z}(u)=v$ and by Hölder's inequality with $p$ as above, we get that

$$
\begin{aligned}
\int_{\mathbb{D}} \mid & K_{r}^{f}(z, u) \mid h(u) d m(u) \\
= & \chi_{\mathbb{D} \backslash r \mathbb{D}}(z) \int_{\mathbb{D}}\left|P\left(\bar{f} \circ \psi_{z}\right)(v)\right|\left|K_{z}\left(\psi_{z}(v)\right)\right| \\
& \times\left(K_{\psi_{z}(v)}\left(\psi_{z}(v)\right)\right)^{\varepsilon}\left|k_{z}(v)\right|^{2} d m(v) \\
= & \chi_{\mathbb{D} \backslash r \mathbb{D}}(z) \int_{\mathbb{D}}\left|P\left(\bar{f} \circ \psi_{z}\right)(v)\right| \frac{1}{1-|z|^{2}} \frac{1}{1-|v|^{2}} \frac{1}{\left(1-\left|\psi_{z}(v)\right|^{2}\right)^{2 \varepsilon-1}} d m(v) \\
\leq & X_{\mathbb{D} \backslash r \mathbb{D}}(z) \frac{1}{1-|z|^{2}}\left(\int_{\mathbb{D}}\left|P\left(\bar{f} \circ \psi_{z}\right)(v)\right|^{p} d m(v)\right)^{1 / p} \\
& \times\left(\int_{\mathbb{D}} \frac{\left(1-|v|^{2}\right)^{-p /(p-1)}}{\left(1-\left|\psi_{z}(v)\right|^{2}\right)^{(2 \varepsilon-1)(p /(p-1))}} d m(v)\right)^{1-1 / p} .
\end{aligned}
$$

To get the last equality, we have used the equation

$$
1-\left|\psi_{z}(v)\right|^{2}=\frac{\left(1-|z|^{2}\right)\left(1-|v|^{2}\right)}{|1-\bar{z} v|^{2}}
$$

Using it one more time and then applying Lemma 3.3 in the last inequality, we get that

$$
\begin{aligned}
\int_{\mathbb{D}}\left|K_{r}^{f}(z, u)\right| h(u) d m(u) \\
\leq \quad X_{\mathbb{D} \backslash r \mathbb{D}}(z) \frac{1}{1-|z|^{2}}\left\|P\left(\bar{f} \circ \psi_{z}\right)\right\|_{p} \\
\quad \times\left(\int_{\mathbb{D}} \frac{1}{\left(1-|v|^{2}\right)^{2 p \varepsilon /(p-1)}} \frac{|1-\bar{z} v|^{2 p(2 \varepsilon-1) /(p-1)}}{\left(1-|z|^{2}\right)^{(2 \varepsilon-1)(p /(p-1))}} d m(v)\right)^{(p-1) / p}
\end{aligned}
$$




$$
\begin{aligned}
= & \chi_{\mathbb{D} \backslash r \mathbb{D}}(z)\left\|T_{\bar{f} \circ \psi_{z}} \mathbf{1}\right\|_{p} \frac{1}{\left(1-|z|^{2}\right)^{2 \varepsilon}} \\
& \times\left(\int_{\mathbb{D}} \frac{\left(1-|v|^{2}\right)^{-2 p \varepsilon /(p-1)}}{|1-\bar{z} v|^{2 p(1-2 \varepsilon) /(p-1)}} d m(v)\right)^{(p-1) / p} \\
\leq & c \sup _{|z| \geq r}\left\|T_{\bar{f} \circ \psi_{z}} \mathbf{1}\right\|_{p} h(z)=c_{2} h(z) .
\end{aligned}
$$

We get the first inequality of the Schur's test in a similar way, noting that

$$
\int_{\mathbb{D}}\left|K_{r}^{f}(z, u)\right| h(z) d m(z)=\chi_{\mathbb{D} \backslash r \mathbb{D}}(z) \int_{\mathbb{D}}\left|T_{f} K_{u}(z)\right|\left(K_{z}(z)\right)^{\varepsilon} d m(z) .
$$

By the same argument as above, the right-hand side is less than or equal to

$$
c \sup _{|u| \geq r}\left\|T_{f \circ \psi_{u}} \mathbf{1}\right\|_{p} h(u) \leq c \sup _{u \in \mathbb{D}}\left\|T_{f \circ \psi_{u}} \mathbf{1}\right\|_{p} h(u)=c_{1} h(u) .
$$

Thus, by Schur's test, $\left\|T^{f}-T_{r}^{f}\right\|^{2} \leq c_{1} c_{2}$, where $c_{1}$ does not depend on $r$, and by Step $4, c_{2} \rightarrow 0$ as $r \rightarrow 1$.

4. Comments and further generalizations. A general problem that motivated the results of this paper is to determine the class of operators $A$ on the Bergman space for which $\widetilde{A}(z) \rightarrow 0$, as $z \rightarrow \partial \mathbb{D}$, implies that $A$ is compact. This class of $C^{\infty}(\mathbb{D})$ functions, vanishing on the boundary, has to be an ideal of the noncommutative algebra of Berezin transform functions on $\mathbb{D}$.

Our result states that Toeplitz operators with $\mathrm{BMO}^{1}$ symbols belong to this ideal. The method of the proof actually yields a stronger result that we state below as Theorem 4.2. The following lemma considers the main technical generalization.

LEMMA 4.1. Let $f$ be in $L^{1}(\mathbb{D})$, let $T_{f}$ be bounded in $L_{a}^{2}(\mathbb{D})$, and let $\tilde{f}(z) \rightarrow 0$ as $z \rightarrow \partial \mathbb{D}$. If there exists $p>3$ such that $\sup _{z \in \mathbb{D}}\left\|T_{f \circ \psi_{z}} \mathbf{1}\right\|_{p}<\infty$, then, for every $q<p$, $\sup _{z \in \mathbb{D}}\left\|T_{f \circ \psi_{z}} \mathbf{1}\right\|_{q}<\infty$ and $\left\|T_{f \circ \psi_{z}} \mathbf{1}\right\|_{q} \rightarrow 0$ as $z \rightarrow \partial \mathbb{D}$.

Proof. Since $q<p$, it follows that $\sup _{z \in \mathbb{D}}\left\|T_{f \circ \psi_{z}} \mathbf{1}\right\|_{q}<\infty$. Let $s=p / q$ and let $t$ be such that $1 / t+1 / s=1$. Then

$$
\begin{aligned}
\left\|T_{f \circ \psi_{z}} \mathbf{1}\right\|_{q}^{q}= & \int_{r \mathbb{D}}\left|T_{f \circ \psi_{z}} \mathbf{1}(\omega)\right|^{q} d m(\omega) \\
& +\int_{\mathbb{D} \backslash r \mathbb{D}}\left|T_{f \circ \psi_{z}} \mathbf{1}(\omega)\right|^{q} d m(\omega) \\
= & I_{1}(z)+I_{2}(z) .
\end{aligned}
$$


By the comment in the proof of Step 2 and since $\tilde{f}(z) \rightarrow 0$ as $\mathrm{Z} \rightarrow \partial \mathbb{D}$, it follows that $T_{f \circ \psi_{z}} \mathbf{1} \rightarrow 0$ weakly as $z \rightarrow \partial \mathbb{D}$, and so $I_{1}(z) \rightarrow 0$ as $z \rightarrow \partial \mathbb{D}$. But

$$
\begin{aligned}
I_{2}(z) & \leq\left(\int_{\mathbb{D} \backslash r \mathbb{D}}\left|T_{f \circ \psi_{z}} \mathbf{1}(\omega)\right|^{q s} d m(\omega)\right)^{1 / s}\left(\int_{\mathbb{D} \backslash r \mathbb{D}} \mathbf{1} d m(\omega)\right)^{1 / t} \\
& \leq\left\|T_{f \circ \psi_{z}} \mathbf{1}\right\|_{p}^{p / s}\left(1-r^{2}\right)^{1 / t}
\end{aligned}
$$

which could be made arbitrarily small independently of $z$ by taking $r$ close enough to 1 . Thus $\left\|T_{f \circ \psi_{z}} \mathbf{1}\right\|_{q} \rightarrow 0$, as $z \rightarrow \partial \mathbb{D}$.

THEOREM 4.2. Let $f$ be in $L^{1}(\mathbb{D})$, let $T_{f}$ be bounded in $L_{a}^{2}(\mathbb{D})$, and suppose that there exists $p>3$ such that

$$
\sup _{z \in \mathbb{D}}\left\|T_{f \circ \psi_{z}} \mathbf{1}\right\|_{p}<\infty, \quad \sup _{z \in \mathbb{D}}\left\|T_{\bar{f} \circ \psi_{z}} \mathbf{1}\right\|_{p}<\infty .
$$

Then $\tilde{f}(z) \rightarrow 0$, as $z \rightarrow \partial \mathbb{D}$, implies that $T_{f}$ is compact on $L_{a}^{2}(\mathbb{D})$.

Proof. By Lemma 4.1, there exists $q>3$ such that $\sup _{z \in \mathbb{D}}\left\|T_{f \circ \psi_{z}} \mathbf{1}\right\|_{q}<\infty$ and $\left\|T_{f \circ \psi_{z}} \mathbf{1}\right\|_{q} \rightarrow 0$ as $z \rightarrow \partial \mathbb{D}$. The same holds for $\left\|T_{\bar{f} \circ \psi_{z}} \mathbf{1}\right\|_{q}$. Closer inspection of the proof of Step 5 shows that this is sufficient to show that $\lim _{r \rightarrow 1} \| T_{r}^{f}-$ $T^{f} \|=0$ (where $T_{r}^{f}$ and $T^{f}$ are defined in Step 5). Hence $T_{f}$ is compact on $L_{a}^{2}(\mathbb{D})$.

It is not known if for every bounded Toeplitz operator $T_{f}, \tilde{f}(z) \rightarrow 0$ as $z \rightarrow \partial \mathbb{D}$, guarantees that $T_{f}$ is compact. Note that $T_{f}$ being bounded on $L_{a}^{2}(\mathbb{D})$ implies that $\sup _{z \in \mathbb{D}}\left\|T_{f \circ \psi_{z}} \mathbf{1}\right\|_{2}<\infty$.

We ask the following question.

QUESTION. For $f$ in $L^{1}(\mathbb{D})$ and $T_{f}$ being bounded on $L_{a}^{2}(\mathbb{D})$, and such that $\sup _{z \in \mathbb{D}}\left\|T_{f \circ \psi_{z}} \mathbf{1}\right\|_{p}<\infty$ and $\sup _{z \in \mathbb{D}}\left\|T_{\bar{f} \circ \psi_{z}} \mathbf{1}\right\|_{p}<\infty$ for some $p>2$, does it follow that $\tilde{f}(z) \rightarrow 0$ as, $z \rightarrow \partial \mathbb{D}$, implies that $T_{f}$ is compact?

ACKNOWLEDGMENT. This work was supported by Natural Science and Engineering Research Council (NSERC) grant.

\section{REFERENCES}

[1] S. Axler and D. Zheng, Compact operators via the Berezin transform, Indiana Univ. Math. J. 47 (1998), no. 2, 387-400.

[2] D. Békollé, C. A. Berger, L. A. Coburn, and K. H. Zhu, BMO in the Bergman metric on bounded symmetric domains, J. Funct. Anal. 93 (1990), no. 2, 310-350.

[3] H. Hedenmalm, B. Korenblum, and K. Zhu, Theory of Bergman Spaces, Graduate Texts in Mathematics, vol. 199, Springer-Verlag, New York, 2000.

[4] H. Li and D. H. Luecking, BMO on strongly pseudoconvex domains: Hankel operators, duality and $\bar{\partial}$-estimates, Trans. Amer. Math. Soc. 346 (1994), no. 2, 661-691. 
[5] D. H. Luecking, Trace ideal criteria for Toeplitz operators, J. Funct. Anal. 73 (1987), no. 2, 345-368.

[6] K. Stroethoff and D. C. Zheng, Toeplitz and Hankel operators on Bergman spaces, Trans. Amer. Math. Soc. 329 (1992), no. 2, 773-794.

[7] J. L. Wang and Z. Wu, Multipliers between BMO spaces on open unit ball, Integral Equations Operator Theory 45 (2003), no. 2, 231-249.

[8] K. H. Zhu, Positive Toeplitz operators on weighted Bergman spaces of bounded symmetric domains, J. Operator Theory 20 (1988), no. 2, 329-357.

[9] _ Operator Theory in Function Spaces, Monographs and Textbooks in Pure and Applied Mathematics, vol. 139, Marcel Dekker, New York, 1990.

[10] N. Zorboska, The Berezin transform and radial operators, Proc. Amer. Math. Soc. 131 (2003), no. 3, 793-800.

Nina Zorboska: Department of Mathematics, University of Manitoba, Winnipeg, Manitobia, Canada R3T 2N2

E-mail address: zorbosk@cc.umani toba.ca 


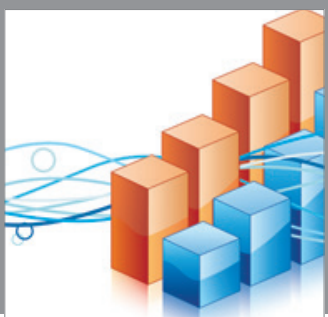

Advances in

Operations Research

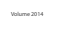

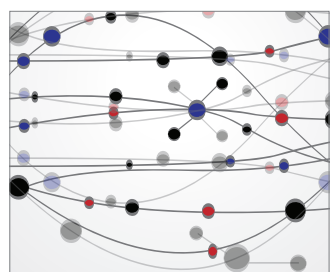

\section{The Scientific} World Journal
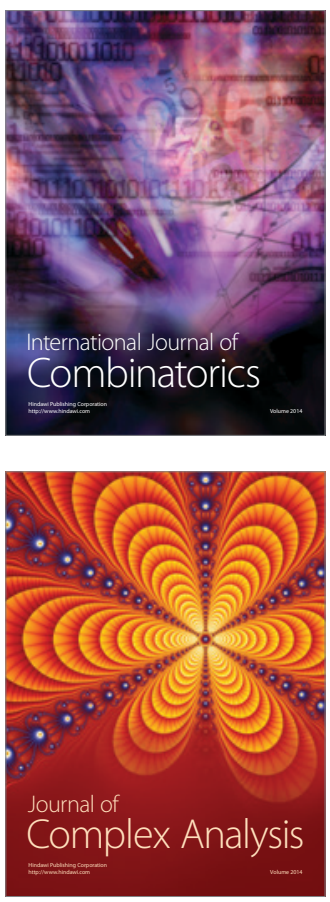

International Journal of

Mathematics and

Mathematical

Sciences
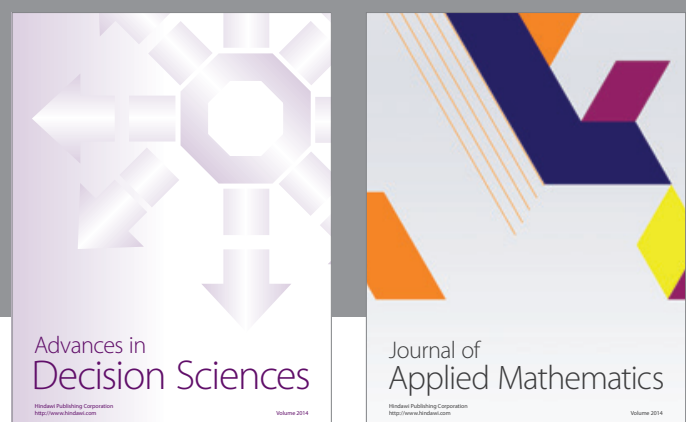

Journal of

Applied Mathematics
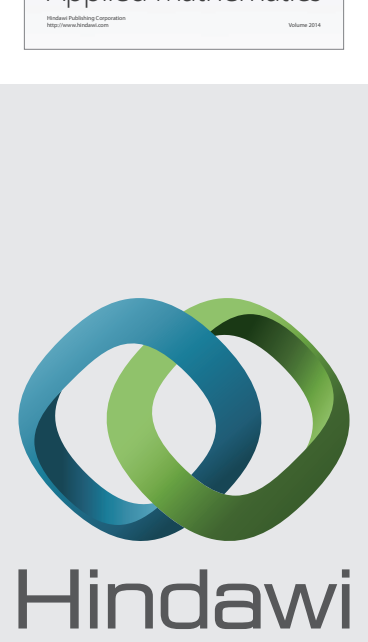

Submit your manuscripts at http://www.hindawi.com
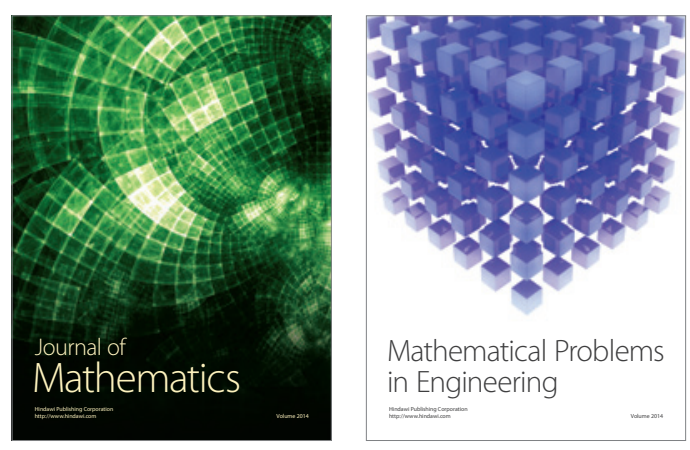

Mathematical Problems in Engineering
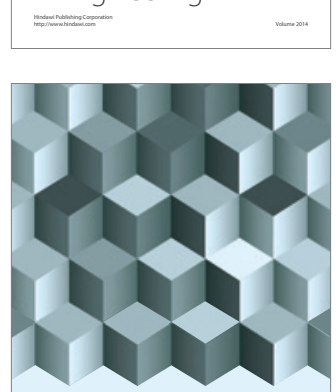

Journal of

Function Spaces
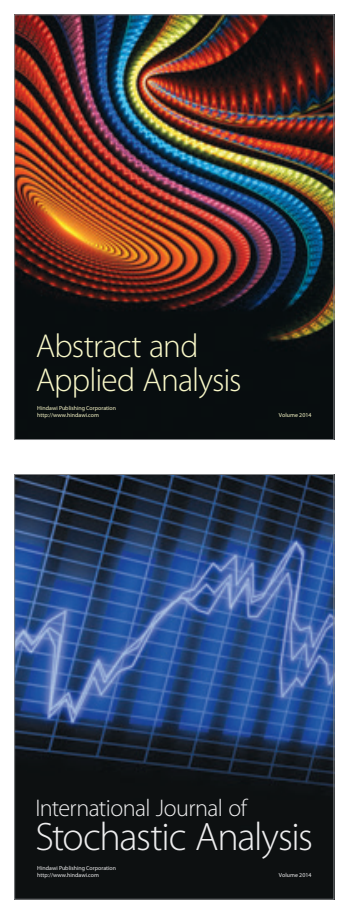

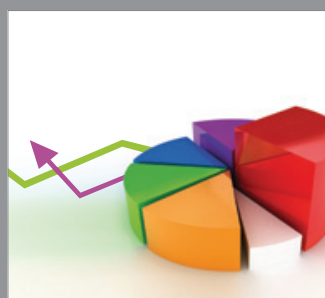

ournal of

Probability and Statistics

Promensencen
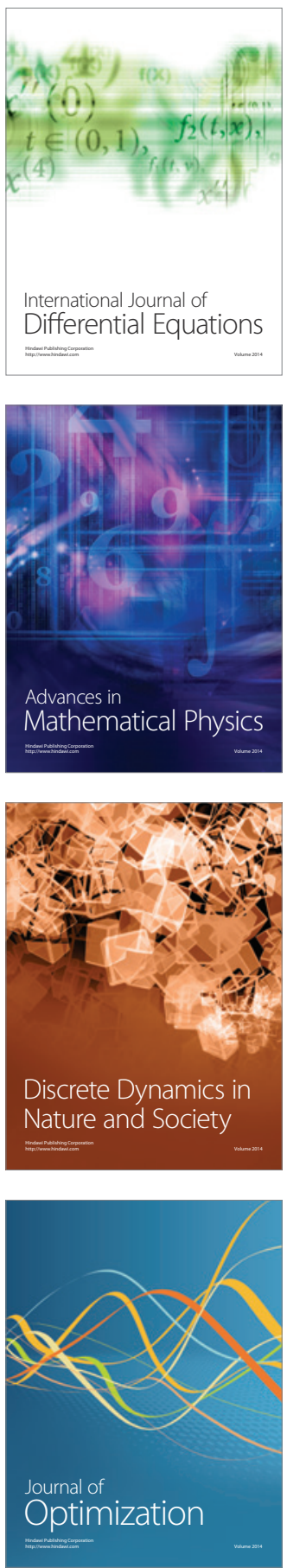